\title{
A Correlative Immunoconfocal and Electron Microscopic Study of Gap Junctions in Interlocking Domains of the Lens
}

\author{
Sondip K. Biswas ${ }^{1}$, Lawrence Brako ${ }^{1}$ and Woo-Kuen Lo ${ }^{1}$ \\ 1. Department of Neurobiology, Morehouse School of Medicine, Atlanta, Georgia
}

Ball-and-sockets and protrusions are specialized interlocking membrane domains between fiber cells of the avascular lenses in all species studied [1, 2]. Although both domains are similar in their shape, surface morphology and are highly enriched with aquaporin-0 water channel protein, our previous studies reveal that ball-and-sockets and protrusions possess important structural and functional differences during fiber cell differentiation and maturation [2, 3]. Specifically, gap junctions are regularly associated with all ball-and-sockets examined in metabolically active young cortical fibers, but not with protrusions. Also, while many ball-and-sockets are distributed primarily on the broad and narrow surfaces of young hexagonal fiber cells, numerous protrusions are located along the angles of the cells throughout the entire lens. It was proposed that the unique ball-and-socket-associated gap junctions may significantly facilitate cell-to-cell communication between young cortical fiber cells since they often protrude deeply into neighboring cells to increase membrane surface areas [2]. In contrast, protrusions are shown to play important interlocking role in maintaining fiber-to-fiber stability during visual accommodation [3].

Since the large number of ball-and-socket domains is distributed only in small superficial cortical regions, it often causes difficulty in conducting a comparative study for the functional roles of gap junction-associated ball-and-socket domains during fiber cell differentiation in the wild-type and genetically-altered mouse models. To facilitate a correlative immunoconfocal and scanning electron microscopic study in identifying the distinct distribution of Cx46 and Cx50 in ball-and-socket domains, we describe here an innovative procedure to prepare SEM imaging from the lens whole-mount samples previously mounted on a glass slide for confocal immunofluorescence labeling. In brief, three edges of the coverslip with mounting medium and nail polish were gently teased away from the slide with a razor blade, but leaving one edge attached to keep the lens whole-mount samples in place. The tissue slide was soaked in PBS for 3-5 min, immersed in 2.5\% glutaraldehyde in 0.1M cacodylate buffer (pH 7.3) for $2 \mathrm{~h}$ at RT, and rinsed in cacodylate buffer. The tissue slide was then fixed in 1\% aqueous $\mathrm{OsO}_{4}$ for 1 $\mathrm{h}$ at $\mathrm{RT}$, and rinsed in $\mathrm{dH}_{2} \mathrm{O}$. The tissue-free coverslip was now loosely floating or detached from the slide. The partially detached tissues were carefully lifted off from the wet slide with a razor blade, and placed in a porous micro-container for conducting critical point drying. Individual whole-mount samples were mounted on a carbon tab/aluminum stub and coated with gold/palladium in a sputter coater. Micrographs were taken with a JEOL 820 scanning electron microscope at $10 \mathrm{kV}$.

By using this procedure, we were able to routinely locate the distinct Cx46 (or Cx50)-associated balland-sockets on the broad sides of cortical fiber cells in the WT lenses by confocal immunolabeling (Fig. 1A-C), and scanning electron microscopy (Fig. 2A). By using freeze-fracture TEM and immunogold labeling on freeze-fracture replica in different experiments, gap junction plaques and Cx46 antibody labeling were found on the ball-and-socket domains (Fig. 2B-C). The results indicate that this novel procedure would be greatly beneficial to our future studies in using the mouse mutant models [4]. 
[1] B Willekens and G Vrensen, Graefes Arch Clin Exp Ophthalmol 219 (1982), p. 112.

[2] SK Biswas et al, Mol Vis 16 (2010), p. 2328.

[3] WK Lo et al, Invest Ophthalmol Vis Sci 55 (2014), p. 1202.

[4] The authors acknowledge funding from the NIH/NEI Grant R01 EY05314 to WKL.

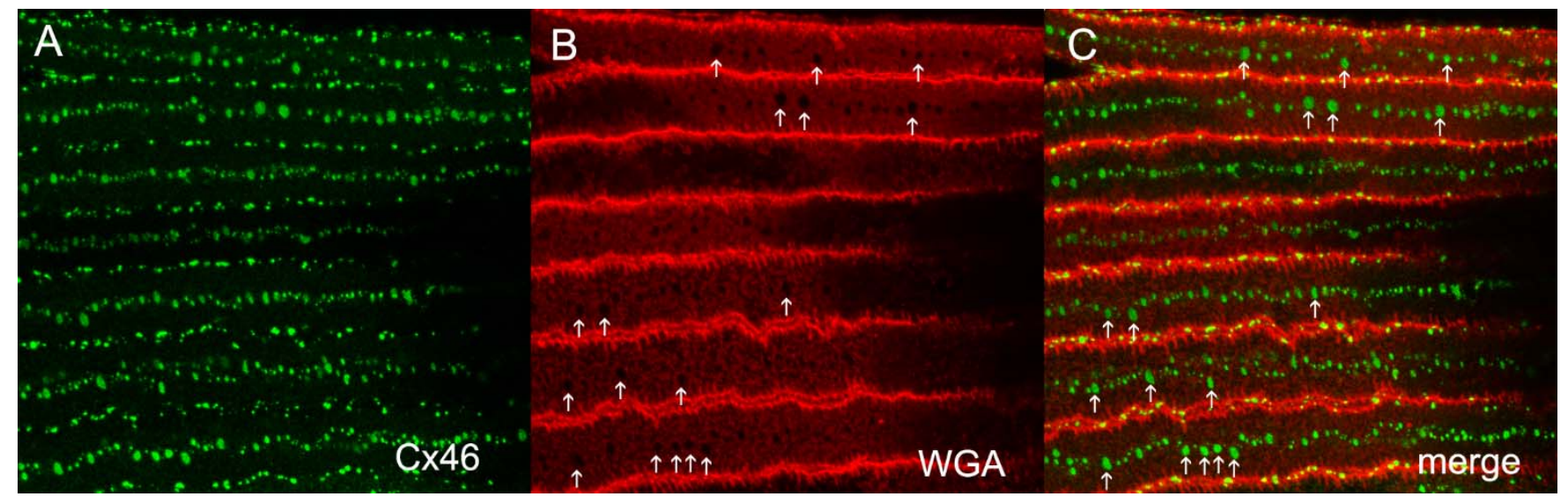

Figure 1. Immunoconfocal labeling shows that $\mathrm{Cx} 46$ antibody is regularly labeled along the broad-sides of superficial fiber cells (A). Wheat germ agglutinin (WGA) was used to label elongated fiber cell membranes (B). While the edges of the cells were heavily stained, many "ball-and-sockets" were displayed as "empty" spaces (arrows) along the broad-side fiber cell surfaces (B). The specific colocalizations between the "sockets" and the distinct, dense Cx46 labeling (arrows) were shown in (C).

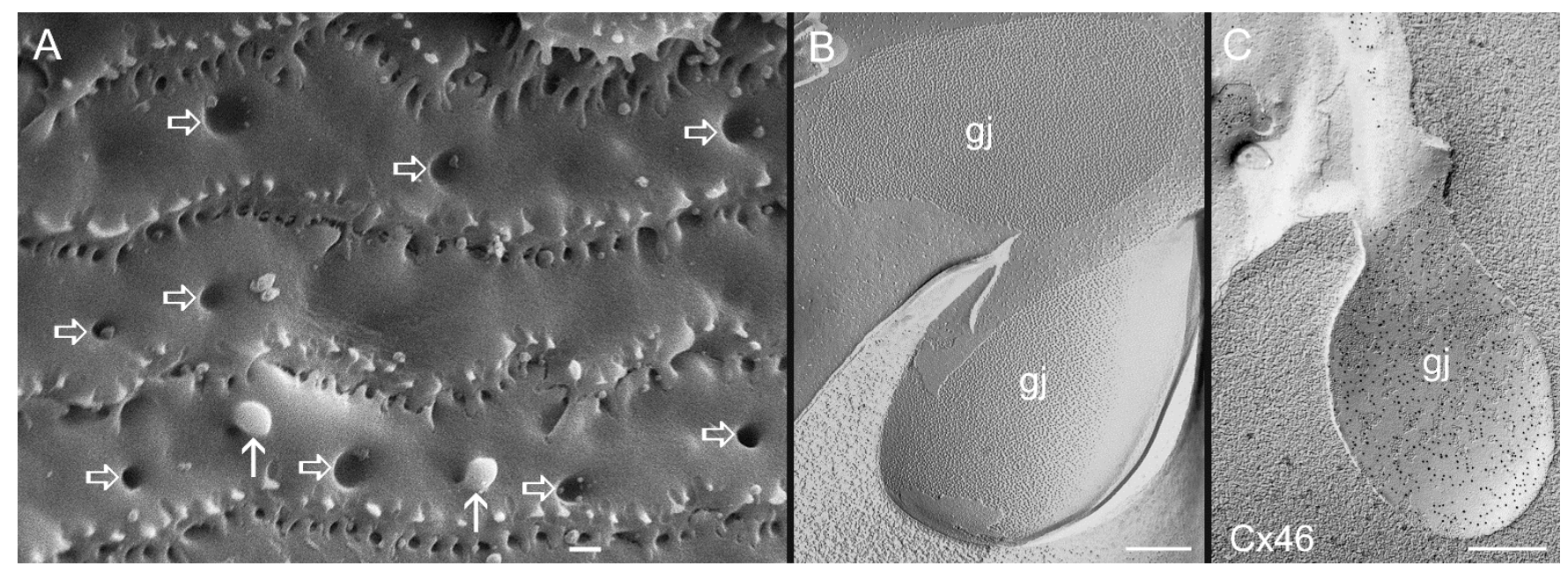

Figure 2. (A) SEM micrograph shows a correlative image of elongated fiber cell surfaces prepared from the previous immunoconfocal samples in Figure 1. Some ball-and-socket domains are indicated by the solid and open arrows. Numerous interlocking protrusions are also shown along the edges between the cells. (B) Freeze-fracture TEM reveals that gap junction plaques are found in both "ball-and-socket" and flat membrane surface. (C) Freeze-fracture immunogold labeling confirms the specific localization of Cx46 antibody (as represented by 10-nm gold particles) in gap junctions of both ball-and-socket and flat membrane. Scale bars: A $=1 \mu \mathrm{m} ; \mathrm{B}=250 \mathrm{~nm} ; \mathrm{C}=500 \mathrm{~nm}$. 